\footnotetext{
${ }^{6}$ More generally, if there is an appreciable spread in the magnitude of the moments of the different clouds, as recent experiments (see Ref. 4) indicate, the saturation magnetization should be equated to $3 k C_{\mathrm{CW}} / \mathrm{Jl}^{*}$ with $\mathfrak{M} *=\left[\mathfrak{M}^{2}\right] /[\mathfrak{M}]$, where the brackets signify averages over the moment distribution.

${ }^{7}$ B. Mozer, D. T. Keating, and S. C. Moss, Phys. Rev. 175, 868 (1968).

${ }^{8} \mathrm{~J}$. W. Cable, E. O. Wollan, and H. R. Child, Phys. Rev. Letters 22, 1256 (1969).

${ }^{9}$ The possibility that many of the polarization clouds are nucleated at $\mathrm{Fe}$ or other magnetic impurity sites is precluded by the low impurity level of $\sim 30-40 \mathrm{ppm}$ in our samples.

${ }^{10}$ N. D. Lang and H. Ehrenreich, Phys. Rev. $\underline{168}, 605$ (1968).
}

\begin{abstract}
${ }^{11} \mathrm{By}$ assuming that the magnitude of each Ni-atom moment in $\mathrm{Ni}-\mathrm{Cu}$ depends only on its local chemical environment, C. G. Robbins, H. Claus, and P. A. Beck [Phys. Rev. Letters 22, 1307 (1969)] obtained an empirical fit to their data for bulk ferromagnetic moment versus alloy composition. However, this assumption ignores purely magnetic correlations, i.e., factors in the local environment that are explicitly magnetic and not uniquely determined by the chemical surroundings. The distance from a Ni-rich nucleating site must be such a factor. Thus, the polarization clouds in $\mathrm{Ni}-\mathrm{Cu}$ are basically analogous in origin to those in $\mathrm{Pd}-\mathrm{Fe}$ [see G. G. Low and T. M. Holden, Proc. Phys. Soc. (London) 89, 119 (1966)].

${ }^{12}$ C. G. Robbins, H. Claus, and P. A. Beck, J. Appl. Phys. 40, 2269 (1969).
\end{abstract}

\title{
ASYMPTOTICALLY CORRECT SHELL MODEL FOR NUCLEAR FISSION*
}

\author{
D. Scharnweber, U. Mosel, and W. Greiner \\ Institut für Theoretische Physik der Universität, Frankfurt am Main, Germany \\ (Received 12 January 1970)
}

\begin{abstract}
A two-center shell model with oscillator potentials, $\overrightarrow{\mathrm{l}} \cdot \overrightarrow{\mathrm{s}}$ forces, and $\overrightarrow{\mathrm{l}}^{2}$ terms is developed. The shell structures of the original spherical nucleus and those of the final fragments are reproduced. For small separation of the two centers the level structure resembles the Nilsson scheme. This two-center shell model might be of importance in problems of nuclear fission.
\end{abstract}

From physical intuition it is evident that it is not possible to describe the process of nuclear fission all the way from the ground state of the fissioning nucleus up to the final stage of two separated fragments by means of a one-center shell model like, e.g., the Nilsson model. It is, instead, essential to allow for a preformation of both final fragments in the deformed shell model. This is in fact an additional degree of freedom. In a recent paper this type of single-particle potential was proposed. ${ }^{1}$ This potential consists of two connected oscillator potentials, including a spin-orbit force and $\overrightarrow{1}^{2}$ term. ${ }^{2}$ Only the angular-momentum-independent terms have been treated in Ref. 1 .

In this paper we report the results of a more realistic calculation including all the $I$-dependent terms and obtain consequently the correct asymptotic single-particle levels for a symmetric two-center shell model.

The Hamiltonian for this potential is

$$
\boldsymbol{H}=-\frac{\hbar^{2}}{2 m} \Delta+\frac{m}{2} \omega_{0}^{2}\left[\rho^{2}+\left(|z|-z_{0}\right)^{2}\right]-\kappa \hbar \omega_{0}^{0}\left\{2 \overrightarrow{\mathrm{s}} \cdot(\nabla V \times \overrightarrow{\mathrm{p}})+\mu\left[(\nabla V \times \overrightarrow{\mathrm{p}})^{2}-\frac{1}{2} N(N+3)\right]\right\},
$$

where $\overrightarrow{\mathrm{p}}$ is the single-particle momentum and $V$ is the momentum-independent part of the potential. It is noticed that $V$ describes two connected oscillators:

$$
\begin{aligned}
V & =(m / 2) \omega_{0}^{2}\left[\left(z-z_{0}\right)^{2}+\rho^{2}\right], z>0 \\
& =(m / 2) \omega_{0}^{2}\left[\left(z+z_{0}\right)^{2}+\rho^{2}\right], \quad z<0
\end{aligned}
$$

while the momentum-dependent terms become

$$
\begin{aligned}
V(\overrightarrow{\mathrm{p}}) & =-\kappa \hbar \omega_{0}^{0}\left\{2 \mathrm{\textrm {I } _ { 1 }} \cdot \overrightarrow{\mathrm{s}}+\mu\left[\overrightarrow{\mathrm{I}}_{1}^{2}-\frac{1}{2} N(N+3)\right]\right\}, \\
& =-\kappa \hbar \omega_{0}^{0}\left\{2 \overrightarrow{\mathrm{I}}_{2} \cdot \overrightarrow{\mathrm{s}}+\mu\left[\overrightarrow{\mathrm{I}}_{2}^{2}-\frac{1}{2} N(N+3)\right]\right\},
\end{aligned}
$$

where $\vec{I}_{1}$ and $\vec{I}_{2}$ describe the angular momenta with respect to the two centers at $z=-z_{0}$ and $z=+z_{0}$, respectively. It follows from (2) and (3) that the Hamiltonian (1) indeed contains for $z_{0}$ $\approx 0$ the case of a spherical Nilsson potential and for $z_{0} \approx R$ ( $R$ being the nuclear radius) the case of two identical and well-separated potentials of the same type. This behavior is due to the special Ansatz for $V$ and is, therefore, automatically present also for the $\vec{p}$-dependent terms in (1). The structure of those latter terms is determined by quite general invariance requirements.

The shape of the two connected (amalgamated) nuclei described by (1) is spherical. It is also 
easy to generalize the shape of the two connected nuclei in Eq. (1) to two connected ellipsoids by choosing different frequencies for the $\rho$ and $z$ degree of freedom. In this way one allows for a deformation of the fragments.

It has been shown in Ref. 1 that the $\vec{p}$-independent terms of the Hamiltonian (1) can be diagonalized nearly analytically by means of a simple matching procedure. In this paper we have used these eigenfunctions as a basis for the diagonalization of the complete Hamiltonian (1). The parameters used here are the usual ones for the neutron spectra in the actinide region: $\kappa=0.0635$ and $\mu=0.325$ while for $\hbar \omega_{0}^{0}$ the value $41 A^{-1 / 3}$ $\mathrm{MeV}$ has been taken. The requirement for volume conservation has been met by an averaging procedure over all equipotential surfaces ${ }^{3}$ thus taking into account automatically the increase of $\hbar \omega_{0}(A)$ to the value $\hbar \omega_{0}(A / 2)$ on the way from the

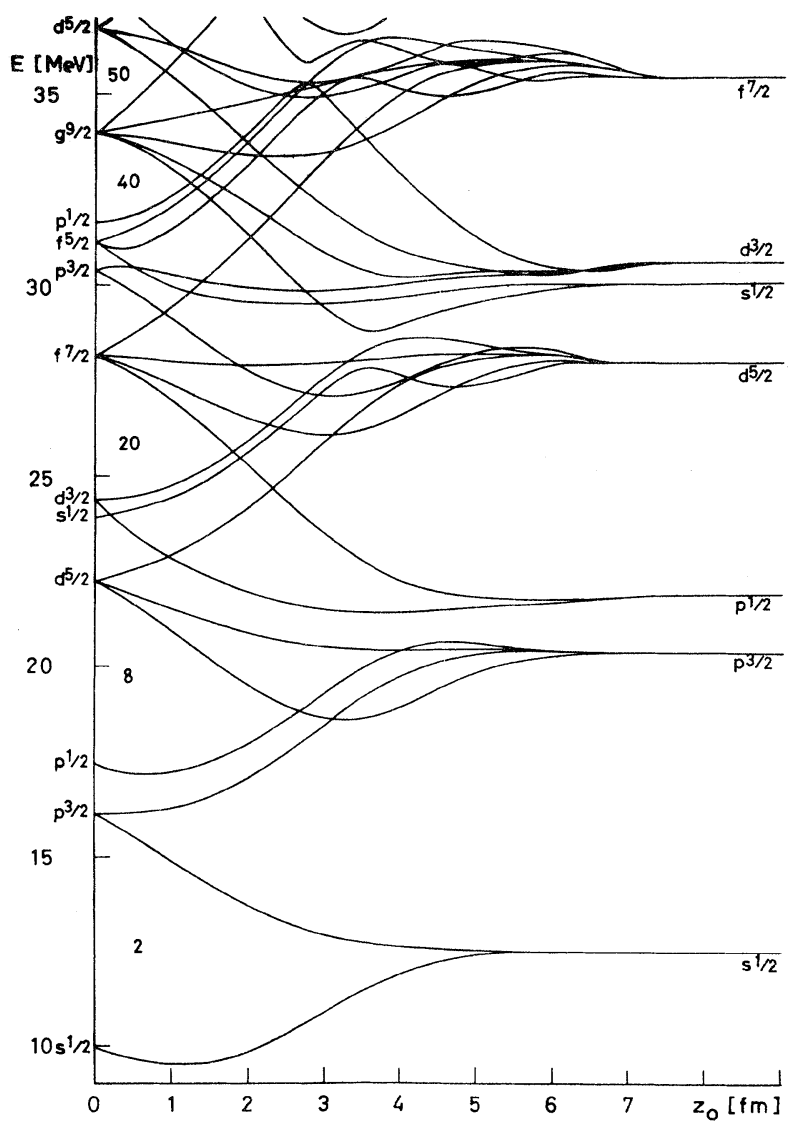

FIG. 1. The complete neutron level scheme of the two-center shell model as a function of the eccentricity parameter $z_{0}$. On the ordinate at the left the spherical shell-model states of the original nucleus are given together with an energy scale while on the righthand side the quantum numbers of the spherical shellmodel states in the fragment nuclei are shown. original spherical nucleus to the two symmetric fragments (i.e., for $z_{0}$ varying between 0 and $\sim R$ ).

The resulting single-particle levels are plotted in Figs. 1 and 2 as a function of the eccentricity parameter $z_{0}$. One sees directly the realistic shell structure of the fissioning nucleus at $z_{0}=0$ with the correct shell closures and magic numbers. It is also noticed that for small eccentricities $z_{0}$ the level scheme resembles the Nilsson model to a very great extent. At the right-hand side of the figure the shell structure of the fragments appears. The larger shell spacing (oscillator spacing) and a double degeneracy of each level according to the presence of two noninteracting identical nuclei is clearly seen. Figures 1 and 2 also show that at larger eccentricities the lowest levels are already completely degenerate, reflecting the fact that a barrier is developing between the two fragments while the higher states near the Fermi surface still show an appreciable splitting. Hence this model leads to a molecular type of state where mainly the "valence nucleons" at the Fermi surface and their shell structure determine the final stages of the fission process. This is indeed well known from the experimental data. ${ }^{4}$

Figure 3 presents the single-particle levels of the Hamiltonian (1) for the actinide region on a larger scale. It is tempting to look for anomalies in the level density, especially for areas of small level density, since it is well known that such regions lead to minima in the total potential-energy surface. ${ }^{5,6}$ In order to be rea-

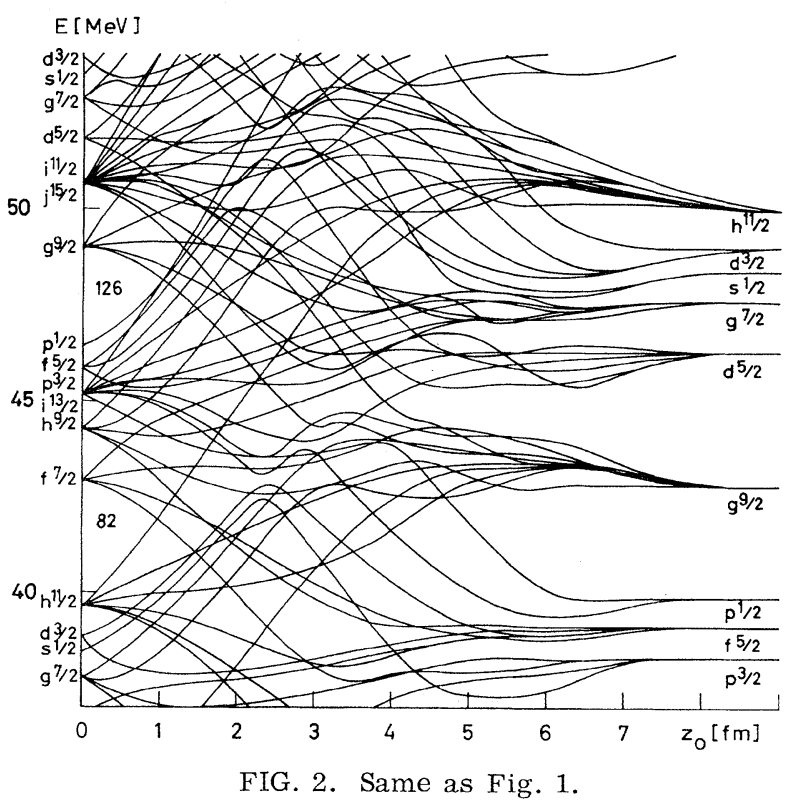




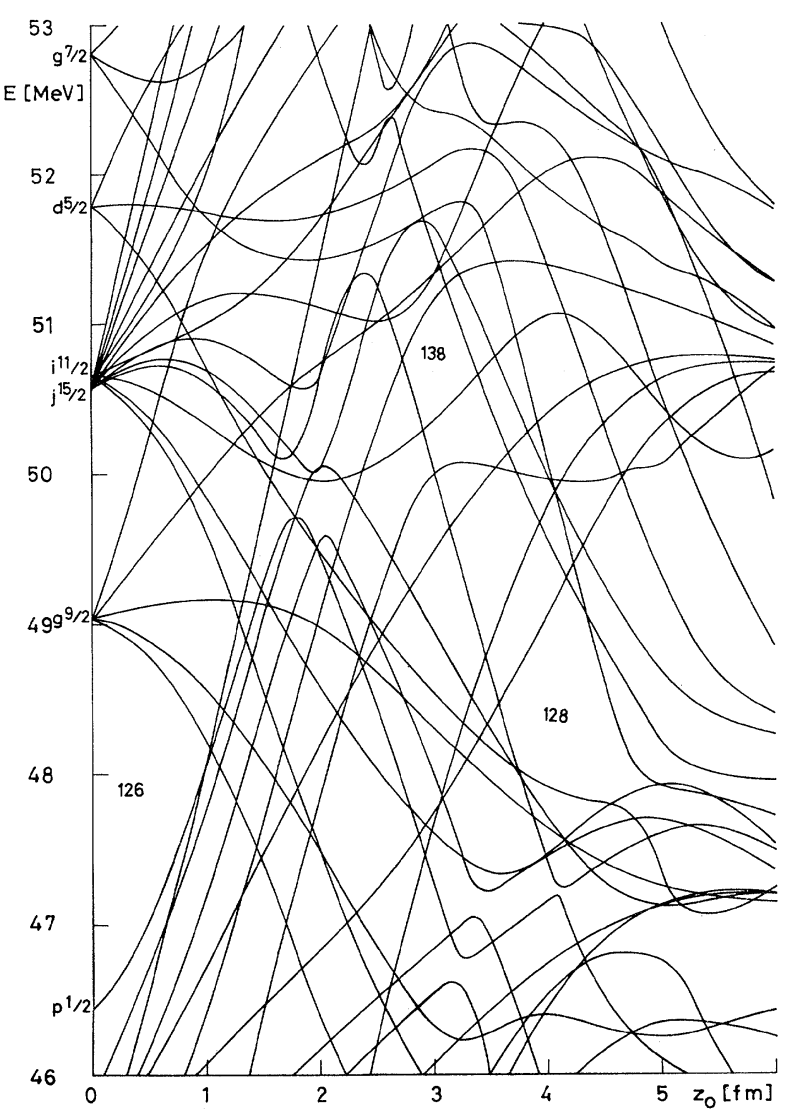

FIG. 3. That part of Fig. 2 which corresponds to the actinide region is plotted on a larger scale. The numbers between the levels give the corresponding neutron occupation numbers.

listic we have to confine ourselves to neutron numbers between $N=120$ and $N \approx 138$ because our potential allows only for symmetric fission and only nuclei with these neutron numbers are known to fission symmetrically. According to Fig. 3 a distinct low-density region appears for the neutron numbers $N=128$ to $N=136$ between $z_{0}=4$ and $z_{0}=3 \mathrm{fm}$. Since the liquid-drop model also shows very flat and broad potential barriers for nuclei in this region ${ }^{7}$ there is some evidence that these deformed shells could lead to fission- isomeric states in these nuclei with an excitation energy of about $10 \mathrm{MeV}$. This point, however, still needs some more detailed investigation.

Summarizing, we may say that we have established a shell model which contains the correct boundary conditions for the fission process and, therefore, is suitable especially for the discussion of single-particle effects at large deformations where already the shell structures of the fragments become important. In particular one may learn from the two-center shell model more about the physical situation at the scission point which has up to now been considered phenomenologically. ${ }^{8}$ A generalization of the potential to asymmetric mass divisions and more refined potentials (Saxon-Woods type) is straightforward and leads to no major difficulties. Such a generalization would especially deepen our understanding of asymmetric fission and its connection to the shell structure of the final fragments.

*Work supported by the Bundesministerium für Wissenschaftliche Forschung and by the Deutsche Forschungsgemeinschaft.

${ }^{1}$ P. Holzer, U. Mosel, and W. Greiner, Nucl. Phys. A138, 241 (1969).

${ }^{2}$ C. Gustafson, I. L. Lamm, B. Nilsson, and S. G. Nilsson, Arkiv Fysik 36, 613 (1967).

${ }^{3}$ This is a rather arbitrary but simple way for volume conservation. Other possibilities including the volume conservation of every equipotential will be extensively discussed in a detailed paper to be published.

${ }^{4}$ E. Konecny and H. W. Schmitt, Phys. Rev. 172, 1213 (1968).

${ }^{5}$ V. M. Strutinsky, Nucl. Phys. A122, 1 (1968).

${ }^{6}$ U. Mosel and W. Greiner, Z. Physik 222, 261 (1969).

${ }^{7}$ H. v. Groote and E. Hilf, Nucl. Phys. $\underline{\mathrm{A} 129}, 513$ (1969).

${ }^{8}$ H. W. Schmitt, Arkiv Fysik 36, 633 (1967), and in Proceedings of the Second Symposium on the Physics and Chemistry of Fission, Vienna, Austria, 1969 (to be published), paper SM-122/122; R. Vandenbosch, Nucl. Phys. 46, 129 (1963); F. Dickmann and K. Dietrich, Nucl. Phys. A129, 241 (1969). 